ESAIM: M2AN 48 (2014) 603-621

DOI: $10.1051 / \mathrm{m} 2 \mathrm{an} / 2013107$
ESAIM: Mathematical Modelling and Numerical Analysis

www.esaim-m2an.org

\title{
A MODIFIED QUASI-BOUNDARY VALUE METHOD FOR THE BACKWARD TIME-FRACTIONAL DIFFUSION PROBLEM
}

\author{
Ting Wei ${ }^{1}$ AND Jun-Gang WANG
}

\begin{abstract}
In this paper, we consider a backward problem for a time-fractional diffusion equation with variable coefficients in a general bounded domain. That is to determine the initial data from a noisy final data. Based on a series expression of the solution, a conditional stability for the initial data is given. Further, we propose a modified quasi-boundary value regularization method to deal with the backward problem and obtain two kinds of convergence rates by using an a priori regularization parameter choice rule and an a posteriori regularization parameter choice rule. Numerical examples in one-dimensional and two-dimensional cases are provided to show the effectiveness of the proposed methods.
\end{abstract}

Mathematics Subject Classification. 35R11, 35R30.

Received November 30, 2012. Revised July 25, 2013.

Published online January 20, 2014.

\section{INTRODUCTION}

The fractional diffusion equations can be used to describe the anomalous diffusion phenomena instead of the classical diffusion procedure and have attracted wide attentions in recent years. The time fractional diffusion equation is deduced by replacing the standard time derivative with a time fractional derivative and can be used to describe superdiffusion and subdiffusion phenomena $[3,20,33,37]$. The direct problems, i.e., initial value problem and initial boundary value problems for the time fractional diffusion equation have been studied extensively in recent years, for instances, on maximum principle [17], on some uniqueness and existence results [16], on numerical solutions by finite element methods [12] and finite difference methods [22,34,41], on exact solutions $[18,19,39]$.

However, for some practical problems, the part of boundary data, or initial data, or diffusion coefficients, or source term may not be given and we want to find them by additional measurement data which will yield to some fractional diffusion inverse problems. The early papers on inverse problems were provided by Murio in $[21,23,24]$ for solving the sideways fractional heat equations by mollification methods. After that, some works have been published. In [4], Cheng et al. considered an inverse problem for determining the order of fractional derivative and diffusion coefficient in a fractional diffusion equation and gave a uniqueness result. In [15], Liu et al. solved a backward problem for the time-fractional diffusion equation by a quasi-reversibility regularization

Keywords and phrases. Backward problem, fractional diffusion equation, modified quasi-boundary value method, convergence analysis, a priori parameter choice, morozov's discrepancy principle.

1 School of Mathematics and Statistics, Lanzhou University, P.R. China. tingwei@lzu.edu.cn 
method. Zheng et al. in $[43,44]$ solved the Cauchy problems for the time fractional diffusion equations on a strip domain by a Fourier regularization and a convolution regularization method. Qian in [30] used a modified kernel method to deal with a sideways fractional equation inverse problem. In [5, 25,32, 38, 42], some inverse source problems were investigated. Furthermore, the nonlinear fractional inverse problems have been considered recently in $[13,31]$. To our knowledge, the research for inverse problems of fractional differential equation is a new topic and do not have too many results now.

In this paper, we consider a backward problem for a time fractional diffusion equation with variable coefficients in a general bounded domain.

Let $\Omega$ be a bounded domain in $\mathbb{R}^{d}$ with sufficient smooth boundary $\partial \Omega$. The backward time-fractional diffusion problem is given by

$$
\begin{cases}D_{t}^{\alpha} u(x, t)=(L u)(x, t), & x \in \Omega, t \in(0, T), 0<\alpha<1, \\ u(x, t)=0, & x \in \partial \Omega, t \in(0, T), \\ u(x, T)=g(x), & x \in \bar{\Omega},\end{cases}
$$

where $D_{t}^{\alpha}$ is the Caputo fractional derivative of order $\alpha(0<\alpha \leq 1)$ defined by

$$
D_{t}^{\alpha} u(x, t)= \begin{cases}\frac{1}{\Gamma(1-\alpha)} \int_{0}^{t} \frac{u_{\tau}(x, \tau)}{(t-\tau)^{\alpha}} \mathrm{d} \tau, & 0<\alpha<1, \\ u_{t}(x, t), & \alpha=1 ;\end{cases}
$$

and $-L$ is a symmetric uniformly elliptic operator defined on $D(-L)=H^{2}(\Omega) \bigcap H_{0}^{1}(\Omega)$ given by

$$
L u(x)=\sum_{i=1}^{d} \frac{\partial}{\partial x_{i}}\left(\sum_{j=1}^{d} a_{i j}(x) \frac{\partial}{\partial x_{j}} u(x)\right)+c(x) u(x), \quad x \in \Omega,
$$

in which the coefficients satisfy

$$
\begin{aligned}
& a_{i j}=a_{j i}, 1 \leq i, j \leq d, a_{i j} \in C^{1}(\bar{\Omega}) \\
& \nu \sum_{i=1}^{d} \xi_{i}^{2} \leq \sum_{i, j=1}^{d} a_{i j}(x) \xi_{i} \xi_{j}, x \in \bar{\Omega}, \xi \in \mathbb{R}^{d}, \text { for a constant } \nu>0 \\
& c(x) \leq 0, x \in \bar{\Omega}, c(x) \in C(\bar{\Omega}) .
\end{aligned}
$$

The backward problem is to find $f(x):=u(x, 0)$ from a noisy final data $g^{\delta}(x)$ which is assumed to satisfy

$$
\left\|g^{\delta}(x)-g(x)\right\| \leq \delta
$$

where $\|\cdot\|$ denotes the $L^{2}(\Omega)$ norm and $\delta>0$ is a noise level.

If $\alpha=1$, the backward problem (1.1) is a classical ill-posed problem and has been studied widely in $[1,6,11,14,35]$ for some contributions. However for the fractional backward problem, to our knowledge, there are very few works, for example, Liu et al. in [15] used a quasi-reversibility method to solve the backward problem in one-dimensional case for special coefficients and Sakamoto et al. gave the existence and uniqueness results in [32]. Zheng et al. in [45] solved a space-fractional backward diffusion problem in a unbounded domain. Comparing with the classical backward problem, due to the nonlocal property of the Caputo fractional derivative, the fractional backward problem (1.1) is much more difficult to solve since a large amount of data at each time layer should be saved. However it is observed that the recovering of initial data is more easy and stable for the fractional backward problem (1.1) than for the classical backward problem $(\alpha=1)$ because the 
fractional problem is a moderately ill-posed problem and the standard backward heat problem is a severely ill-posed problem.

The quasi-boundary value method, also called non-local boundary value problem method in [10], is a regularization technique by replacing the final condition or boundary condition by a new approximate condition such that the new problem is well-posed. This method has been used to solve some inverse problems for parabolic equations $[2,7,10,11]$, hype-parabolic equations [36], elliptic equations [8,9].

The usual quasi-boundary value method to deal with the backward problem (1.1) is to perturb the final condition to form an approximate regularized problem

$$
\begin{cases}D_{t}^{\alpha} v(x, t)=(L v)(x, t), & x \in \Omega, t \in(0, T), \\ v(x, t)=0, & x \in \partial \Omega, t \in(0, T), \\ v(x, T)+\mu v(x, 0)=g^{\delta}(x), & x \in \bar{\Omega}\end{cases}
$$

where $\mu$ plays a role of regularization parameter. It can be proved that the best convergence rate for $\| v(x, 0)-$ $u(x, 0) \|$ is $O\left(\delta^{1 / 2}\right)$ under an a priori choice of regularization parameter and an a priori bound assumption. One can see [40] for the same problem with $L=\triangle$ and some numerical examples on square domains with a special fractional order $\alpha=1 / 2$.

In this study, we propose a modified version of quasi-boundary value method to solve the backward problem (1.1), i.e. replacing the final condition with a perturbed condition containing the values of $L u$ at $t=0$ as

$$
v(x, T)-\mu(L v)(x, 0)=g^{\delta}(x), \quad x \in \bar{\Omega} .
$$

For this modification, we can obtain a better convergence rate $O\left(\delta^{2 / 3}\right)$ under an a priori choice of regularization parameter and the same a priori bound assumption compared with one in [40]. Meanwhile by using Morozov's discrepancy principle for choosing the regularization parameter, we can also obtain a convergence rate which is not available in the usual quasi-boundary value method (1.8).

In this paper we solve directly the regularized problem by a finite difference method for one-dimensional case and a finite element method for two-dimensional case. Our numerical methods do not need to compute the Mittag-Leffler function and eigenfunctions for the second order elliptic operator which is more efficient for the problem with variable coefficients in a general bounded domain than the method in [40]. Note that numerical implementations for the backward problem of fractional order process in high dimensional case with variable coefficients are very few by now. This paper is a very beginning research.

The paper is organized as follows. In Section 2, we give some preliminary results. The ill-posedness of the backward problem (1.1) and a conditional stability are provided in Section 3. In Section 4, we propose a modified quasi-boundary value method and give two convergence estimates under an a priori assumption for the exact solution and two regularization parameter choice rules. Numerical implementation methods are given in Section 5. Finally two numerical examples in one-dimensional and two-dimensional cases respectively are tested in Section 6 for showing that the proposed methods are effective and stable. A brief conclusion is given in Section 7.

\section{PRELIMINARIES}

Throughout this paper, we use the following definition and lemmas, see [27].

Definition 2.1. The Mittag-Leffler function is

$$
E_{\alpha, \beta}(z)=\sum_{k=0}^{\infty} \frac{z^{k}}{\Gamma(\alpha k+\beta)}, \quad z \in \mathbb{C},
$$

where $\alpha>0$ and $\beta \in \mathbb{R}$ are arbitrary constants. 
Lemma 2.2. Let $\lambda>0$, then we have

$$
D_{t}^{\alpha} E_{\alpha, 1}\left(-\lambda t^{\alpha}\right)=-\lambda E_{\alpha, 1}\left(-\lambda t^{\alpha}\right), \quad t>0, \quad 0<\alpha<1 .
$$

Lemma 2.3 ([29]). For $0<\alpha<1, \eta>0$, we have $0 \leq E_{\alpha, 1}(-\eta)<1$. Moreover, $E_{\alpha, 1}(-\eta)$ is completely monotonic, that is

$$
(-1)^{n} \frac{d^{n}}{d \eta^{n}} E_{\alpha, 1}(-\eta) \geq 0
$$

We provide the following lemmas which will be used in the proofs of convergence estimates.

Lemma 2.4. For any $\lambda_{n}$ satisfying $\lambda_{n} \geq \lambda_{1}>0$, there exist positive constants $\underline{C}, \bar{C}$ depending on $\alpha, T, \lambda_{1}$ such that

$$
\frac{\underline{C}}{\lambda_{n}} \leq E_{\alpha, 1}\left(-\lambda_{n} T^{\alpha}\right) \leq \frac{\bar{C}}{\lambda_{n}}
$$

Proof. From Corollary 2.2 in [15], we know there exist positive constants $C_{-}, C_{+}$such that

$$
E_{\alpha, 1}\left(-\lambda_{n} T^{\alpha}\right) \leq \frac{C_{+}}{1+\lambda_{n} T^{\alpha}} \leq \frac{C_{+}}{T^{\alpha}} \frac{1}{\lambda_{n}}
$$

and

$$
E_{\alpha, 1}\left(-\lambda_{n} T^{\alpha}\right) \geq \frac{C_{-}}{1+\lambda_{n} T^{\alpha}} \geq \frac{C_{-}}{\frac{\lambda_{n}}{\lambda_{1}}+\lambda_{n} T^{\alpha}}=\frac{C_{-}}{\frac{1}{\lambda_{1}}+T^{\alpha}} \frac{1}{\lambda_{n}} .
$$

Denote $\bar{C}=\frac{C_{+}}{T^{\alpha}}$ and $\underline{C}=\frac{C_{-}}{\lambda_{1}+T^{\alpha}}$, then the proof is completed.

Lemma 2.5. For constants $p>0, \mu>0, \beta>0, s \geq \lambda_{1}>0$, we have

$$
F(s)=\frac{\mu s^{2-\frac{p}{2}}}{\mu s^{2}+\beta} \leq \begin{cases}C_{1} \mu^{\frac{p}{4}}, & 0<p<4, \\ C_{2} \mu, & p \geq 4,\end{cases}
$$

where $C_{1}=C_{1}(p, \beta)>0, C_{2}=C_{2}\left(p, \beta, \lambda_{1}\right)>0$ are independent of $s$.

Proof. If $0<p<4$, then we have $\lim _{s \rightarrow 0} F(s)=\lim _{s \rightarrow \infty} F(s)=0$, thus

$$
F(s) \leq \sup _{s \in(0,+\infty)} F(s) \leq F\left(s_{0}\right),
$$

where $s_{0} \in(0,+\infty)$ such that $F^{\prime}\left(s_{0}\right)=0$. It is easy to prove that $s_{0}=\left(\frac{\beta(4-p)}{p \mu}\right)^{\frac{1}{2}}>0$, thus we have

$$
F(s) \leq \frac{\left(\frac{\beta(4-p)}{p}\right)^{\frac{4-p}{4}}\left(\frac{1}{\mu}\right)^{\frac{4-p}{4}} \mu}{\frac{\beta(4-p)}{p}+\beta}=: C_{1}(p, \beta) \mu^{\frac{p}{4}} .
$$

If $p \geq 4$, then for $s \geq \lambda_{1}>0$, we have

$$
F(s) \leq \frac{\mu}{\mu s^{2}+\beta} \frac{1}{s^{\frac{p}{2}-2}} \leq \frac{\mu}{\beta} \frac{1}{\lambda_{1}^{\frac{p}{2}-2}}=: C_{2}\left(p, \beta, \lambda_{1}\right) \mu .
$$

Lemma 2.6. For constants $p>0, \mu>0, \beta>0, s \geq \lambda_{1}>0$, we have

$$
G(s)=\frac{\mu s^{1-\frac{p}{2}}}{\mu s^{2}+\beta} \leq \begin{cases}C_{3} \mu^{\frac{2+p}{4}}, & 0<p<2, \\ C_{4} \mu, & p \geq 2,\end{cases}
$$

where $C_{3}=C_{3}(p, \beta)>0, C_{4}=C_{4}\left(p, \beta, \lambda_{1}\right)>0$ are independent of $s$. 
Proof. If $0<p<2$, then we have $\lim _{s \rightarrow 0} G(s)=\lim _{s \rightarrow \infty} G(s)=0$, thus we know

$$
G(s) \leq \sup _{s \in(0,+\infty)} G(s) \leq G\left(s_{0}\right)
$$

where $s_{0} \in(0,+\infty)$ such that $G^{\prime}\left(s_{0}\right)=0$. It is easy to prove that $s_{0}=\left(\frac{\beta(2-p)}{(2+p) \mu}\right)^{\frac{1}{2}}>0$, thus we have

$$
G(s) \leq G\left(s_{0}\right)=\frac{\left(\frac{\beta(2-p)}{2+p}\right)^{\frac{2-p}{4}}\left(\frac{1}{\mu}\right)^{\frac{2-p}{4}} \mu}{\frac{\beta(2-p)}{(2+p)}+\beta}=: C_{3}(p, \beta) \mu^{\frac{2+p}{4}} .
$$

If $p \geq 2$, then we have

$$
G(s) \leq \frac{\mu}{\mu s^{2}+\beta} \frac{1}{s^{\frac{p}{2}-1}} \leq \frac{\mu}{\beta} \frac{1}{\lambda_{1}^{\frac{p}{2}-1}}=: C_{4}\left(p, \beta, \lambda_{1}\right) \mu .
$$

\section{ILL-POSEDNESS AND A CONDITIONAL STABILITY FOR THE BACKWARD PROBLEM}

Denote the eigenvalues of the operator $-L$ as $\lambda_{n}$ and the corresponding eigenfunctions as $\varphi_{n}(x) \in$ $H^{2}(\Omega) \bigcap H_{0}^{1}(\Omega)$, then we have

$$
L \varphi_{n}=-\lambda_{n} \varphi_{n}
$$

Since $-L$ is a symmetric uniformly elliptic operator, counting according to the multiplicities, we can assume

$$
0<\lambda_{1} \leq \lambda_{2} \leq \ldots \leq \lambda_{n} \leq \ldots, \quad \lim _{n \rightarrow \infty} \lambda_{n}=+\infty
$$

and $\left\{\varphi_{n}\right\}_{n=1}^{\infty}$ is the orthonormal basis in $L^{2}(\Omega)$.

Define

$$
D\left((-L)^{\gamma}\right)=\left\{\psi \in L^{2}(\Omega) ; \sum_{n=1}^{\infty} \lambda_{n}^{2 \gamma}\left|\left(\psi, \varphi_{n}\right)\right|^{2}<\infty\right\},
$$

where $(\cdot, \cdot)$ is the inner product in $L^{2}(\Omega)$, then $D\left((-L)^{\gamma}\right)$ is a Hilbert space with the norm

$$
\|\psi\|_{D\left((-L)^{\gamma}\right)}=\left(\sum_{n=1}^{\infty} \lambda_{n}^{2 \gamma}\left|\left(\psi, \varphi_{n}\right)\right|^{2}\right)^{\frac{1}{2}} .
$$

From Theorem 4.1 in [32], we know there exists a unique weak solution $u \in C\left([0, T] ; L^{2}(\Omega)\right) \cap$ $C\left((0, T] ; H^{2}(\Omega) \bigcap H_{0}^{1}(\Omega)\right)$ to problem (1.1) if $g \in H^{2}(\Omega) \cap H_{0}^{1}(\Omega)$. However the noisy measurement data $g^{\delta}$ of $g$ is usually not in $H^{2}(\Omega) \bigcap H_{0}^{1}(\Omega)$ and even the difference $\left\|g^{\delta}-g\right\|$ is small, the solutions of problem (1.1) to $g^{\delta}$ and $g$ may have a big difference. That means the backward problem is ill-posed, see the following statement. We will provide a conditional stability in Theorem 3.1. In this paper, we focus mainly on the regularization method for solving (1.1).

By the separation of variables and Lemma 2.2, we know the formal solution for (1.1) can be expressed by

$$
u(x, t)=\sum_{n=1}^{\infty}\left(u(x, 0), \varphi_{n}\right) E_{\alpha, 1}\left(-\lambda_{n} t^{\alpha}\right) \varphi_{n}(x) .
$$

Denote $f_{n}=\left(f, \varphi_{n}\right), g_{n}=\left(g, \varphi_{n}\right)$, then let $t=T$, we have

$$
g(x)=\sum_{n=1}^{\infty} f_{n} E_{\alpha, 1}\left(-\lambda_{n} T^{\alpha}\right) \varphi_{n}(x),
$$


and

$$
g_{n}=f_{n} E_{\alpha, 1}\left(-\lambda_{n} T^{\alpha}\right) .
$$

If we take $g=g_{k}(x)=E_{\alpha, 1}\left(-\lambda_{k} T^{\alpha}\right) \varphi_{k}(x)$ in (1.1), then by Lemma 2.4, we know $\left\|g_{k}\right\|=E_{\alpha, 1}\left(-\lambda_{k} T^{\alpha}\right) \leq$ $\bar{C} / \lambda_{k} \rightarrow 0$, as $k \rightarrow \infty$, however the corresponding initial data are $f_{k}(x)=\varphi_{k}(x)$, since $\left\|f_{k}\right\|=1$, we know the backward problem (1.1) is ill-posed.

To find $f(x)$, we just need to solve the following first kind Fredholm integral equation

$$
(K f)(x)=\int_{\Omega} k(x, \xi) f(\xi) \mathrm{d} \xi=g(x), \quad x \in \Omega,
$$

where the kernel is

$$
k(x, \xi)=\sum_{n=1}^{\infty} E_{\alpha, 1}\left(-\lambda_{n} T^{\alpha}\right) \varphi_{n}(x) \varphi_{n}(\xi) .
$$

We give a conditional stability in the following theorem.

Theorem 3.1. Let $f(x):=u(x, 0) \in D\left((-L)^{\frac{p}{2}}\right)$ satisfy an a priori bound condition

$$
\|f\|_{D\left((-L)^{\frac{p}{2}}\right.} \leq E, \quad p>0
$$

then we have

$$
\|f\| \leq C_{5} E^{\frac{2}{p+2}}\|g\|^{\frac{p}{p+2}}, \quad p>0,
$$

where $C_{5}=\underline{C}^{-\frac{p}{p+2}}$ is a constant depending on $\alpha, T, p, \lambda_{1}$.

Proof. From (3.6) and the Hölder inequality, we have

$$
\begin{aligned}
\|f\|^{2} & =\sum_{n=1}^{\infty} f_{n}^{2}=\sum_{n=1}^{\infty} \frac{g_{n}^{2}}{E_{\alpha, 1}^{2}\left(-\lambda_{n} T^{\alpha}\right)} \\
& =\sum_{n=1}^{\infty} \frac{g_{n}^{\frac{4}{p+2}}}{E_{\alpha, 1}^{2}\left(-\lambda_{n} T^{\alpha}\right)} g_{n}^{\frac{2 p}{p+2}} \\
& \leq\left(\sum_{n=1}^{\infty} \frac{g_{n}^{2}}{E_{\alpha, 1}^{p+2}\left(-\lambda_{n} T^{\alpha}\right)}\right)^{\frac{2}{p+2}}\left(\sum_{n=1}^{\infty} g_{n}^{2}\right)^{\frac{p}{p+2}} .
\end{aligned}
$$

Applying Lemma 2.4, we obtain

$$
\begin{aligned}
\sum_{n=1}^{\infty} \frac{g_{n}^{2}}{E_{\alpha, 1}^{p+2}\left(-\lambda_{n} T^{\alpha}\right)} & \leq \sum_{n=1}^{\infty} \frac{g_{n}^{2}}{E_{\alpha, 1}^{2}\left(-\lambda_{n} T^{\alpha}\right)}\left(\frac{\lambda_{n}}{\underline{C}}\right)^{p} \\
& =\sum_{n=1}^{\infty} f_{n}^{2} \lambda_{n}^{p} \underline{C}^{-p}=\|f\|_{D\left((-L)^{\frac{p}{2}}\right)}^{2} \underline{C}^{-p} .
\end{aligned}
$$

Combining (3.10)-(3.11), we get

$$
\|f\|^{2} \leq \underline{C}^{-\frac{2 p}{p+2}}\|f\|_{D\left((-L)^{\frac{p}{2}}\right)}^{\frac{4}{p+2}}\|g\|^{\frac{2 p}{p+2}} .
$$

The proof is completed.

Remark 3.2. The conditional stability result (3.9) is Hölder type, but by using the same a priori bound condition (3.8), the Hölder type estimate for the case of $\alpha=1$ is impossible. This is a big difference between the classical backward problem and fractional backward problem. It indicates that the reconstruction of initial data is more stable for the fractional order equation than the case of $\alpha=1$. 


\section{A MOdified QUASI-BOUNDARY VALUE METHOD AND CONVERGENCE RATES}

In this section, we propose a modified quasi-boundary value method to solve problem (1.1) and give two convergence estimates under an priori regularization parameter choice rule and an a posteriori regularization parameter choice rule, respectively.

Let $u_{\mu}^{\delta}(x, t)$ be the solution of the following regularized problem

$$
\begin{cases}D_{t}^{\alpha} v(x, t)=(L v)(x, t), & x \in \Omega, t \in(0, T), \\ v(x, t)=0, & x \in \partial \Omega, t \in(0, T), \\ v(x, T)-\mu(L v)(x, 0)=g^{\delta}(x), & x \in \bar{\Omega}\end{cases}
$$

where $\mu>0$ is a regularization parameter. In following subsections, we will prove that $u_{\mu}^{\delta}(x, 0)$ is a regularized solution for the backward problem (1.1).

By the separation of variables, we know $u_{\mu}^{\delta}(x, t)$ has the following form

$$
u_{\mu}^{\delta}(x, t)=\sum_{n=1}^{\infty} C_{n} E_{\alpha, 1}\left(-\lambda_{n} t^{\alpha}\right) \varphi_{n}(x) .
$$

From (4.1c), we get

$$
C_{n} E_{\alpha, 1}\left(-\lambda_{n} T^{\alpha}\right)+\mu C_{n} \lambda_{n}=g_{n}^{\delta}
$$

where $g_{n}^{\delta}=\left(g^{\delta}, \varphi_{n}\right)$. Thus, $C_{n}=\frac{g_{n}^{\delta}}{E_{\alpha, 1}\left(-\lambda_{n} T^{\alpha}\right)+\mu \lambda_{n}}$. Substituting $C_{n}$ into (4.2), we get

$$
u_{\mu}^{\delta}(x, t)=\sum_{n=1}^{\infty} \frac{g_{n}^{\delta}}{E_{\alpha, 1}\left(-\lambda_{n} T^{\alpha}\right)+\mu \lambda_{n}} E_{\alpha, 1}\left(-\lambda_{n} t^{\alpha}\right) \varphi_{n}(x) .
$$

Denote

$$
u_{\mu}(x, t)=\sum_{n=1}^{\infty} \frac{g_{n}}{E_{\alpha, 1}\left(-\lambda_{n} T^{\alpha}\right)+\mu \lambda_{n}} E_{\alpha, 1}\left(-\lambda_{n} t^{\alpha}\right) \varphi_{n}(x) .
$$

In the following, we give two convergence estimates for $\left\|u_{\mu}^{\delta}(x, 0)-u(x, 0)\right\|$ by using an a priori and an a posteriori choice rule for the regularization parameter.

\subsection{Convergence estimate under an a priori regularization parameter choice rule}

Theorem 4.1. Suppose the a priori condition (3.8) and the noise Assumption (1.7) hold, then,

(1) If $0<p<4$ and choose $\mu=\left(\frac{\delta}{E}\right)^{\frac{4}{p+2}}$, we have a convergence estimate

$$
\left\|u_{\mu}^{\delta}(x, 0)-u(x, 0)\right\| \leq\left(\frac{1}{2 \sqrt{\underline{C}}}+C_{1}\right) E^{\frac{2}{p+2}} \delta^{\frac{p}{p+2}} ;
$$

(2) If $p \geq 4$ and choose $\mu=\left(\frac{\delta}{E}\right)^{\frac{2}{3}}$, we have a convergence estimate

$$
\left\|u_{\mu}^{\delta}(x, 0)-u(x, 0)\right\| \leq\left(\frac{1}{2 \sqrt{\underline{C}}}+C_{2}\right) E^{\frac{1}{3}} \delta^{\frac{2}{3}},
$$

where $C_{1}, C_{2}$ are positive constants depending on $p, \alpha, T$ and $\lambda_{1}$. 
Proof. By the triangle inequality, we know

$$
\left\|u_{\mu}^{\delta}(x, 0)-u(x, 0)\right\| \leq\left\|u_{\mu}^{\delta}(x, 0)-u_{\mu}(x, 0)\right\|+\left\|u_{\mu}(x, 0)-u(x, 0)\right\| .
$$

We firstly give an estimate for the first term. From (4.3), (4.4) and (1.7), we have

$$
\left\|u_{\mu}^{\delta}(x, 0)-u_{\mu}(x, 0)\right\|^{2}=\sum_{n=1}^{\infty}\left(\frac{g_{n}^{\delta}-g_{n}}{E_{\alpha, 1}\left(-\lambda_{n} T^{\alpha}\right)+\mu \lambda_{n}}\right)^{2} \leq \delta_{n}^{2}\left(\sup _{n} A(n)\right)^{2},
$$

where

$$
A(n)=\frac{1}{E_{\alpha, 1}\left(-\lambda_{n} T^{\alpha}\right)+\mu \lambda_{n}}
$$

By Lemma 2.4, we get

$$
A(n) \leq \frac{1}{\frac{C}{\lambda_{n}}+\mu \lambda_{n}} \leq \frac{1}{2 \sqrt{\underline{C} \mu}}
$$

Substituting it into (4.6), we obtain

$$
\left\|u_{\mu}^{\delta}(x, 0)-u_{\mu}(x, 0)\right\| \leq \frac{1}{2 \sqrt{\underline{C}}} \frac{\delta}{\sqrt{\mu}} .
$$

Now we estimate the second term in (4.5). By (4.4), we can deduce that

$$
\begin{aligned}
u_{\mu}(x, 0)-u(x, 0) & =\sum_{n=1}^{\infty}\left(\frac{g_{n}}{E_{\alpha, 1}\left(-\lambda_{n} T^{\alpha}\right)+\mu \lambda_{n}}-\frac{g_{n}}{E_{\alpha, 1}\left(-\lambda_{n} T^{\alpha}\right)}\right) \varphi_{n}(x) \\
& =\sum_{n=1}^{\infty}-\frac{g_{n}}{E_{\alpha, 1}\left(-\lambda_{n} T^{\alpha}\right)} \frac{\mu \lambda_{n}}{E_{\alpha, 1}\left(-\lambda_{n} T^{\alpha}\right)+\mu \lambda_{n} \varphi_{n}(x)} .
\end{aligned}
$$

Applying the a priori bound condition (3.8), we obtain

$$
\begin{aligned}
\left\|u_{\mu}(x, 0)-u(x, 0)\right\|^{2} & =\sum_{n=1}^{\infty}\left(\frac{g_{n}}{E_{\alpha, 1}\left(-\lambda_{n} T^{\alpha}\right)}\right)^{2} \lambda_{n}^{p}\left(\frac{\mu \lambda_{n}}{E_{\alpha, 1}\left(-\lambda_{n} T^{\alpha}\right)+\mu \lambda_{n}}\right)^{2} \frac{1}{\lambda_{n}^{p}} \\
& \leq E^{2}\left(\sup _{n} B(n)\right)^{2},
\end{aligned}
$$

where

$$
B(n)=\frac{\mu \lambda_{n}^{1-\frac{p}{2}}}{E_{\alpha, 1}\left(-\lambda_{n} T^{\alpha}\right)+\mu \lambda_{n}} .
$$

By Lemmas 2.4 and 2.5, we have

$$
\begin{aligned}
B(n) & \leq \frac{\mu \lambda_{n}^{1-\frac{p}{2}}}{\mu \lambda_{n}+\frac{\underline{C}}{\lambda_{n}}} \leq \frac{\mu \lambda_{n}^{2-\frac{p}{2}}}{\mu \lambda_{n}^{2}+\underline{C}} \\
& \leq \begin{cases}C_{1}(p, \underline{C}) \mu^{\frac{p}{4}}, & 0<p<4, \\
C_{2}\left(p, \underline{C}, \lambda_{1}\right) \mu, & p \geq 4 .\end{cases}
\end{aligned}
$$

Substituting the above inequality into (4.9) and using (4.7), we obtain

$$
\left\|u_{\mu}^{\delta}(x, 0)-u(x, 0)\right\| \leq \frac{1}{2 \sqrt{\underline{C}}} \frac{\delta}{\sqrt{\mu}}+ \begin{cases}C_{1} E \mu^{\frac{p}{4}}, & 0<p<4 \\ C_{2} E \mu, & p \geq 4\end{cases}
$$


Choose the regularization parameter $\mu$ by

$$
\mu= \begin{cases}\left(\frac{\delta}{E}\right)^{\frac{4}{p+2}}, & 0<p<4 \\ \left(\frac{\delta}{E}\right)^{\frac{2}{3}}, & p \geq 4\end{cases}
$$

then, we have

$$
\left\|u_{\mu}^{\delta}(x, 0)-u(x, 0)\right\| \leq \begin{cases}\left(\frac{1}{2 \sqrt{\underline{C}}}+C_{1}\right) E^{\frac{2}{p+2}} \delta^{\frac{p}{p+2}}, & 0<p<4, \\ \left(\frac{1}{2 \sqrt{\underline{C}}}+C_{2}\right) E^{\frac{1}{3}} \delta^{\frac{2}{3}}, & p \geq 4 .\end{cases}
$$

The proof is completed.

\subsection{Convergence estimate under an a posteriori regularization parameter choice rule}

In this subsection, we use an a posterior regularization parameter choice, i.e., Morozov's discrepancy principle to choose the regularization parameter $\mu$ in (4.2). Based on the conditional stability estimate in Theorem 3.1, we can obtain a convergence rate for the regularized solution (4.2).

Morozov's discrepancy principle for our case is to find $\mu$ such that

$$
\left\|K u_{\mu}^{\delta}(x, 0)-g^{\delta}(x)\right\|=\tau \delta
$$

where $\tau>1$ is a constant. According to the following lemma, we know there exists a unique solution for (4.14) if $\left\|g^{\delta}\right\|>\tau \delta>0$.

Lemma 4.2. Denote $\rho(\mu)=\left\|K u_{\mu}^{\delta}(x, 0)-g^{\delta}(x)\right\|$, then the following results hold:

(a) $\rho(\mu)$ is a continuous function;

(b) $\lim _{\mu \rightarrow 0} \rho(\mu)=0$;

(c) $\lim _{\mu \rightarrow+\infty} \rho(\mu)=\left\|g^{\delta}(x)\right\|$;

(d) $\rho(\mu)$ is a strictly increasing function over $(0, \infty)$.

Proof. The proofs are straightforward results by the expression of

$$
\rho(\mu)=\left(\sum_{n=1}^{\infty}\left(\frac{\mu \lambda_{n}}{E_{\alpha, 1}\left(-\lambda_{n} T^{\alpha}\right)+\mu \lambda_{n}}\right)^{2}\left(g_{n}^{\delta}\right)^{2}\right)^{\frac{1}{2}} .
$$

Theorem 4.3. Suppose the a priori condition (3.8) and the noise assumption (1.7) hold, and there exists $\tau>1$ such that $\left\|g^{\delta}\right\|>\tau \delta>0$. The regularization parameter $\mu>0$ is chosen by Morozov's discrepancy principle (4.14). Then

(1) If $0<p<2$, we have a convergence estimate

$$
\left\|u_{\mu}^{\delta}(x, 0)-u(x, 0)\right\| \leq\left(C_{5}(\tau+1)^{\frac{p}{p+2}}+\frac{1}{2 \sqrt{\underline{C}}}\left(\frac{C_{3}}{\tau-1}\right)^{\frac{2}{2+p}}\right) E^{\frac{2}{p+2}} \delta^{\frac{p}{p+2}} ;
$$

(2) If $p \geq 2$, we have a convergence estimate

$$
\left\|u_{\mu}^{\delta}(x, 0)-u(x, 0)\right\| \leq\left(C_{5}(\tau+1)^{\frac{p}{p+2}}+\frac{1}{2 \sqrt{\underline{C}}}\left(\frac{C_{4}}{\tau-1}\right)^{\frac{1}{2}}\right) E^{\frac{1}{2}} \delta^{\frac{1}{2}},
$$

where $C_{3}, C_{4}, C_{5}$ are positive constants depending on $p, \alpha, T$ and $\lambda_{1}$. 
Proof. Similar to (4.5), we have

$$
\left\|u_{\mu}^{\delta}(x, 0)-u(x, 0)\right\| \leq\left\|u_{\mu}^{\delta}(x, 0)-u_{\mu}(x, 0)\right\|+\left\|u_{\mu}(x, 0)-u(x, 0)\right\| .
$$

We firstly give an estimate for the second term. From (4.8), we know

$$
\begin{aligned}
& K\left(u_{\mu}(x, 0)-u(x, 0)\right)=\sum_{n=1}^{\infty} g_{n} \frac{-\mu \lambda_{n}}{E_{\alpha, 1}\left(-\lambda_{n} T^{\alpha}\right)+\mu \lambda_{n}} \varphi_{n}(x) \\
& =\sum_{n=1}^{\infty}\left(g_{n}-g_{n}^{\delta}\right) \frac{-\mu \lambda_{n}}{E_{\alpha, 1}\left(-\lambda_{n} T^{\alpha}\right)+\mu \lambda_{n}} \varphi_{n}(x)+\sum_{n=1}^{\infty} g_{n}^{\delta} \frac{-\mu \lambda_{n}}{E_{\alpha, 1}\left(-\lambda_{n} T^{\alpha}\right)+\mu \lambda_{n}} \varphi_{n}(x) .
\end{aligned}
$$

Using (1.7) and (4.14), we get

$$
\left\|K\left(u_{\mu}(x, 0)-u(x, 0)\right)\right\| \leq \delta+\tau \delta=(\tau+1) \delta .
$$

Applying the a priori bound condition for $u(x, 0)$, we obtain

$$
\begin{aligned}
\left\|u_{\mu}(x, 0)-u(x, 0)\right\|_{D\left((-L)^{\frac{p}{2}}\right)} & =\left(\sum_{n=1}^{\infty}\left(\frac{g_{n}}{E_{\alpha, 1}\left(-\lambda_{n} T^{\alpha}\right)} \frac{-\mu \lambda_{n}}{E_{\alpha, 1}\left(-\lambda_{n} T^{\alpha}\right)+\mu \lambda_{n}} \lambda_{n}^{\frac{p}{2}}\right)^{2}\right)^{\frac{1}{2}} \\
& \leq\left(\sum_{n=1}^{\infty}\left(\frac{g_{n}}{E_{\alpha, 1}\left(-\lambda_{n} T^{\alpha}\right)}\right)^{2} \lambda_{n}^{p}\right)^{\frac{1}{2}} \leq E .
\end{aligned}
$$

By Theorem 3.1, we deduce that

$$
\left\|u_{\mu}(x, 0)-u(x, 0)\right\| \leq C_{5}(\tau+1)^{\frac{p}{p+2}} E^{\frac{2}{p+2}} \delta^{\frac{p}{p+2}}, \quad \forall p>0 .
$$

Now we give the bound for the first term. Similar to (4.7), we have

$$
\left\|u_{\mu}^{\delta}(x, 0)-u_{\mu}(x, 0)\right\| \leq \frac{1}{2 \sqrt{\underline{C}}} \frac{\delta}{\sqrt{\mu}} .
$$

From (4.14), there holds

$$
\begin{aligned}
\tau \delta & =\left\|\sum_{n=1}^{\infty} \frac{\mu \lambda_{n}}{E_{\alpha, 1}\left(-\lambda_{n} T^{\alpha}\right)+\mu \lambda_{n}} g_{n}^{\delta} \varphi_{n}(x)\right\| \\
& \leq\left\|\sum_{n=1}^{\infty} \frac{\mu \lambda_{n}}{E_{\alpha, 1}\left(-\lambda_{n} T^{\alpha}\right)+\mu \lambda_{n}}\left(g_{n}^{\delta}-g_{n}\right) \varphi_{n}(x)\right\|+\left\|\sum_{n=1}^{\infty} \frac{\mu \lambda_{n}}{E_{\alpha, 1}\left(-\lambda_{n} T^{\alpha}\right)+\mu \lambda_{n}} g_{n} \varphi_{n}(x)\right\| \\
& \leq \delta+J .
\end{aligned}
$$

Using again the a priori bound condition for $u(x, 0)$, we obtain

$$
\begin{aligned}
J & =\left\|\sum_{n=1}^{\infty} \frac{\mu \lambda_{n} E_{\alpha, 1}\left(-\lambda_{n} T^{\alpha}\right)}{E_{\alpha, 1}\left(-\lambda_{n} T^{\alpha}\right)+\mu \lambda_{n}} \frac{1}{\lambda_{n}^{\frac{p}{2}}} \frac{g_{n}}{E_{\alpha, 1}\left(-\lambda_{n} T^{\alpha}\right)} \lambda_{n}^{\frac{p}{2}} \varphi_{n}(x)\right\| \\
& \leq \sup _{n} C(n) .
\end{aligned}
$$

From Lemma 2.4, we have

$$
C(n)=\frac{\mu \lambda_{n} E_{\alpha, 1}\left(-\lambda_{n} T^{\alpha}\right)}{E_{\alpha, 1}\left(-\lambda_{n} T^{\alpha}\right)+\mu \lambda_{n}} \frac{1}{\lambda_{n}^{\frac{p}{2}}} \leq \frac{\mu \lambda_{n} \frac{\bar{C}}{\lambda_{n}}}{\frac{\underline{\underline{C}}}{\lambda_{n}}+\mu \lambda_{n}} \frac{1}{\lambda_{n}^{\frac{p}{2}}} \leq \frac{\bar{C} \mu \lambda_{n}^{1-\frac{p}{2}}}{\underline{C}+\mu \lambda_{n}^{2}} .
$$


By Lemma 2.6, (4.24) becomes

$$
C(n) \leq \begin{cases}C_{3}(p, \underline{C}, \bar{C}) \mu^{\frac{2+p}{4}}, & 0<p<2, \\ C_{4}\left(p, \underline{C}, \bar{C}, \lambda_{1}\right) \mu, & p \geq 2 .\end{cases}
$$

Combining (4.21)-(4.25), we obtain

$$
(\tau-1) \delta \leq \begin{cases}C_{3} E \mu^{\frac{2+p}{4}}, & 0<p<2, \\ C_{4} E \mu, & p \geq 2 .\end{cases}
$$

This yields

$$
\frac{1}{\mu} \leq \begin{cases}\left(\frac{C_{3}}{\tau-1}\right)^{\frac{4}{2+p}}\left(\frac{E}{\delta}\right)^{\frac{4}{2+p}}, & 0<p<2 \\ \frac{C_{4}}{\tau-1} \frac{E}{\delta}, & p \geq 2 .\end{cases}
$$

Substituting (4.27) into (4.20), we get

$$
\begin{gathered}
\left\|u_{\mu}^{\delta}(x, 0)-u(x, 0)\right\| \leq C_{5}(\tau+1)^{\frac{p}{p+2}} E^{\frac{2}{p+2}} \delta^{\frac{p}{p+2}} \\
+ \begin{cases}\frac{1}{2 \sqrt{\underline{C}}}\left(\frac{C_{3}}{\tau-1}\right)^{\frac{2}{2+p}} E^{\frac{2}{2+p}} \delta^{\frac{p}{2+p}}, & 0<p<2, \\
\frac{1}{2 \sqrt{\underline{C}}}\left(\frac{C_{4}}{\tau-1}\right)^{\frac{1}{2}} E^{\frac{1}{2}} \delta^{\frac{1}{2}}, & p \geq 2 .\end{cases}
\end{gathered}
$$

This ends the proof.

\section{NumERICAL IMPLEMENTATIONS}

Since the analytic solution of problem (1.1) is difficult to obtain, we construct the final data $g(x)$ by solving the following forward problem

$$
\begin{cases}D_{t}^{\alpha} u(x, t)=(L u)(x, t), & x \in \Omega, t \in(0, T), 0<\alpha<1 \\ u(x, t)=0, & x \in \partial \Omega, t \in(0, T), \\ u(x, 0)=f(x), & x \in \bar{\Omega} .\end{cases}
$$

with the given data $f(x)$.

Numerical schemes for the forward problem (5.1) and the regularized problem (4.1a)-(4.1c) are sketched as follows.

\subsection{One-dimensional case}

Without loss of generality, we assume $\Omega=(0,1)$. Take the grid sizes for time and space variable in the finite difference algorithm are $\Delta t=\frac{T}{N}$ and $\Delta x=\frac{1}{M}$, respectively. The grid points in the time interval $[0, T]$ are labeled $t_{n}=n \Delta t, n=0,1, \ldots, N$, and the grid points in the space interval $[0,1]$ are $x_{i}=i \Delta x, i=0,1,2, \ldots, M$. The approximate values of function $u$ at the grid points are denoted $u_{i}^{n} \approx u\left(x_{i}, t_{n}\right)$. 
The time-fractional derivative is approximated by

$$
D_{t}^{\alpha} u\left(x_{i}, t_{n}\right) \approx \frac{(\Delta t)^{-\alpha}}{\Gamma(2-\alpha)} \sum_{j=0}^{n-1} b_{j}\left(u_{i}^{n-j}-u_{i}^{n-j-1}\right)
$$

for $i=1, \ldots, M-1, n=1, \ldots, N$ where $b_{j}=(j+1)^{1-\alpha}-j^{1-\alpha}$. This scheme was used in $[26,46]$.

We use the scheme

$$
L u\left(x_{i}, t_{n}\right) \approx \frac{1}{(\Delta x)^{2}}\left(a_{i+\frac{1}{2}} u_{i+1}^{n}-\left(a_{i+\frac{1}{2}}+a_{i-\frac{1}{2}}\right) u_{i}^{n}+a_{i-\frac{1}{2}} u_{i-1}^{n}\right)+c\left(x_{i}\right) u_{i}^{n}
$$

for $i=1, \ldots, M-1, n=1, \ldots, N$ to approach the value of $L u$ at point $\left(x_{i}, t_{n}\right)$ where $a_{i+\frac{1}{2}}=a\left(x_{i+\frac{1}{2}}\right)$ with $x_{i+\frac{1}{2}}=\left(x_{i}+x_{i+1}\right) / 2$.

By the initial condition in (5.1), it is easy to get a numerical solution for forward problem (5.1) from the finite difference scheme

$$
\frac{(\Delta t)^{-\alpha}}{\Gamma(2-\alpha)} \sum_{j=0}^{n-1} b_{j}\left(u_{i}^{n-j}-u_{i}^{n-j-1}\right)=\frac{1}{(\Delta x)^{2}}\left(a_{i+\frac{1}{2}} u_{i+1}^{n}-\left(a_{i+\frac{1}{2}}+a_{i-\frac{1}{2}}\right) u_{i}^{n}+a_{i-\frac{1}{2}} u_{i-1}^{n}\right)+c\left(x_{i}\right) u_{i}^{n} .
$$

For the regularized problem (4.1a)-(4.1c), we discretize equation (4.1a) by the finite difference scheme mentioned above. The condition (4.1c) is approximated by

$$
v_{i}^{N}-\mu\left(\frac{1}{(\Delta x)^{2}}\left(a_{i+\frac{1}{2}} v_{i+1}^{0}-\left(a_{i+\frac{1}{2}}+a_{i-\frac{1}{2}}\right) v_{i}^{0}+a_{i-\frac{1}{2}} v_{i-1}^{0}\right)+c\left(x_{i}\right) v_{i}^{0}\right)=g^{\delta}\left(x_{i}\right)
$$

for $i=1, \ldots, M-1$.

\subsection{Two-dimensional case}

For the forward problem, we compute firstly some eigenfunctions of operator $-L$ and then compute the Fourier coefficients of $f(x)$, by using the formula (3.5) with a suitable truncation to get the function $g(x)$ as the "exact" final data. In our computations, we use the matlab command pdeeig to generate eigenfunctions.

To solve the regularized problem (4.1a)-(4.1c) in two dimensional case, we use the finite difference scheme (5.2) to approach the time fractional derivative and then employ a finite element method to discretize the resulted elliptic problem at each time step. By the nonlocal boundary condition (4.1c), we can finally deduce a linear system of equations for the initial data on mesh nodes. The detail is given in the following.

Denote $v^{n}(x)=v\left(x, t_{n}\right)$. By the finite difference scheme (5.2), we can deduce the following Dirichlet problems for elliptic equations

$$
\left\{\begin{array}{l}
v^{1}(x)-r L v^{1}(x)=v^{0}(x), \quad x \in \Omega \\
v^{1}(x)=0, \quad x \in \partial \Omega
\end{array}\right.
$$

and

$$
\left\{\begin{array}{l}
v^{n}(x)-r L v^{n}(x)=\sum_{j=0}^{n-2}\left(b_{j}-b_{j+1}\right) v^{n-j-1}(x)+b_{n-1} v^{0}(x), x \in \Omega, \\
v^{n}(x)=0, \quad x \in \partial \Omega
\end{array}\right.
$$

for $n=2, \ldots, N$, where $r=\Gamma(2-\alpha) \Delta t^{\alpha}$. 
Let $x_{j}, j=1,2, \ldots, m$ be the mesh nodes located in $\Omega$ and $\psi_{j}$ be the corresponding finite element basis functions. Denote $V^{n}=\left(v\left(x_{1}, t_{n}\right), \ldots, v\left(x_{m}, t_{n}\right)\right)^{T}$, then by the standard finite element procedure, we can deduce the following linear equations for (5.4) and (5.5) as

$$
(M+r K+r Q) V^{1}=M V^{0},
$$

and

$$
(M+r K+r Q) V^{n}=\sum_{j=0}^{n-2}\left(b_{j}-b_{j+1}\right) M V^{n-j-1}+b_{n-1} M V^{0},
$$

where $M=\left(\left(\psi_{i}, \psi_{j}\right)\right)_{m \times m}, K=\left(\sum_{k, l=1}^{2}\left(a_{k l} \partial_{x_{k}} \psi_{i}, \partial_{x_{l}} \psi_{j}\right)\right)_{m \times m}, Q=\left(\left(-c \psi_{i}, \psi_{j}\right)\right)_{m \times m}$ are mass matrix and stiff matrixes in which $(\cdot, \cdot)$ is the $L^{2}$ inner product. By a recursion process, we can find a relationship between $V^{0}$ and $V^{N}$ with

$$
V^{N}=A V^{0}
$$

where $A$ is a matrix. The nonlocal boundary condition (4.1c) in finite element scheme becomes

$$
M V^{N}+\mu(K+Q) V^{0}=M G^{\delta},
$$

where $G^{\delta}=\left(g^{\delta}\left(x_{1}\right), \ldots, g^{\delta}\left(x_{m}\right)\right)^{T}$.

Substituting (5.8) into (5.9), we can get

$$
(M A+\mu(K+Q)) V^{0}=M G^{\delta} .
$$

Solving the above linear system of equations, we finally obtain the regularized solution $u_{\mu}^{\delta}(x, 0)=\sum_{j=1}^{m} V_{j}^{0} \psi_{j}(x)$.

\section{NumericAl EXPERIMENTS}

In this section, we present some numerical results for two examples in one-dimensional and two-dimensional cases to show the effectiveness and stability of our proposed method.

The noisy data is generated by adding a random perturbation, i.e.,

$$
g^{\delta}=g+\varepsilon g \cdot(2 \cdot \operatorname{rand}(\operatorname{size}(g))-1) .
$$

The corresponding noise level is calculated by $\delta=\varepsilon\|g\|$.

To show the accuracy of numerical solution, we compute the approximate $L^{2}$ error denoted by

$$
e(f, \varepsilon)=\left\|u(x, 0)-u_{\mu}^{\delta}(x, 0)\right\|
$$

and the approximate relative error in $L^{2}$ norm denoted by

$$
e_{r}(f, \varepsilon)=\left\|u(x, 0)-u_{\mu}^{\delta}(x, 0)\right\| /\|u(x, 0)\| .
$$

To verify the convergence rate, we use the following definition

$$
\text { Convergence Order }=\log _{2} \frac{e(f, 2 \varepsilon)}{e(f, \varepsilon)} \text {. }
$$

In our numerical experiments, we always fix $T=1$. For computing the Mittag-Leffler function, we need a better algorithm, e.g. see [28]. In general, the a priori bound $E$ is difficult to obtain, thus we only give the numerical results by using the a posteriori parameter choice rule which is independent of $E$. The regularization parameter is chosen by (4.14) with $\tau=1.1$. Here we use the matlab command fzero to find $\mu$. 


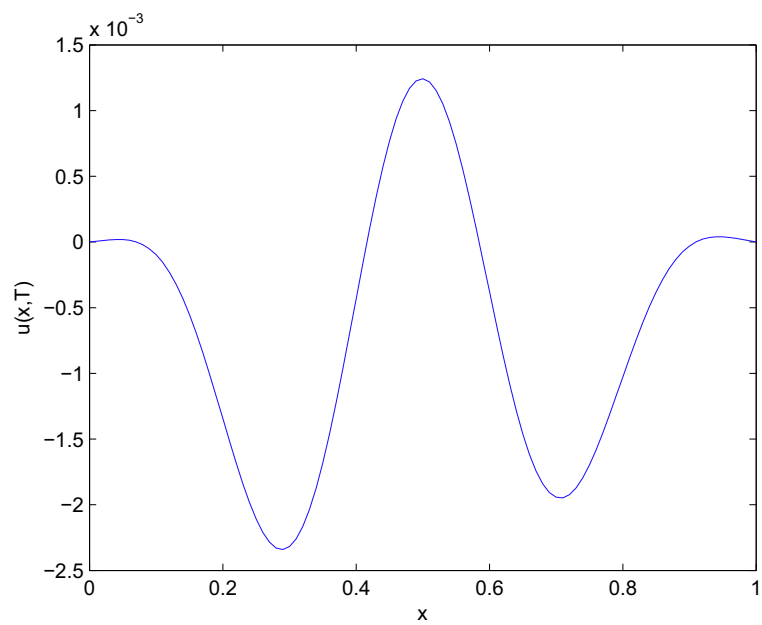

(a) $\alpha=0.2$

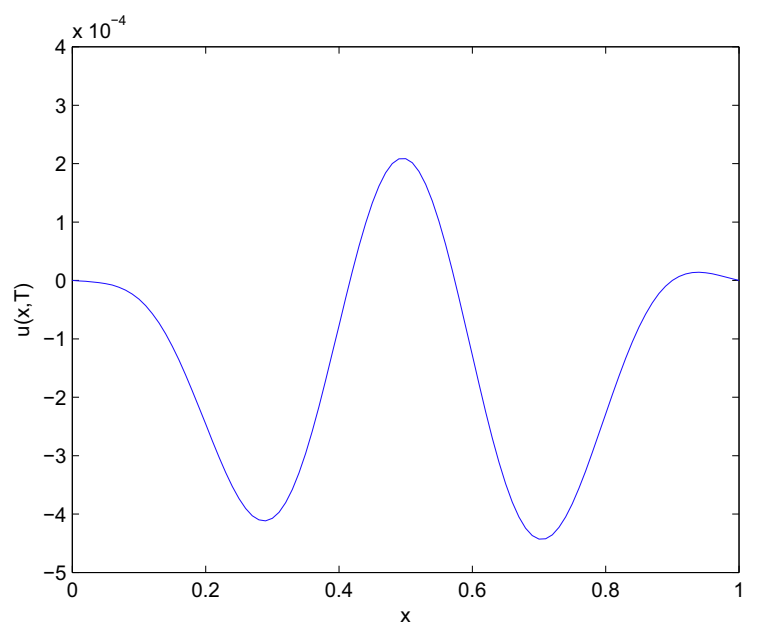

(b) $\alpha=0.8$

Figure 1. The final data for Example 1.

TABle 1. Numerical results of Example 1 for different $\alpha$ with $\varepsilon=0.01$.

\begin{tabular}{ccccccc}
\hline$\alpha$ & 0.05 & 0.1 & 0.3 & 0.7 & 0.9 & 0.95 \\
\hline$e(f, 0.01)$ & 0.0124 & 0.0120 & 0.0109 & 0.0096 & 0.0093 & 0.0092 \\
$e_{r}(f, 0.01)$ & 0.0366 & 0.0368 & 0.0375 & 0.0395 & 0.0407 & 0.0411 \\
\hline
\end{tabular}

TABLE 2. Numerical results of Example 1 for different $\varepsilon$ with $\alpha=0.6$.

\begin{tabular}{ccccccccc}
\hline$\varepsilon$ & 0.0005 & 0.001 & 0.002 & 0.004 & 0.008 & 0.016 & 0.032 & 0.064 \\
\hline$e(f, \varepsilon)$ & 0.0028 & 0.0036 & 0.0047 & 0.0064 & 0.0088 & 0.0125 & 0.0187 & 0.0296 \\
$e_{r}(f, \varepsilon)$ & 0.0113 & 0.0141 & 0.0186 & 0.0252 & 0.0349 & 0.0497 & 0.0741 & 0.1171 \\
\hline Order & & 0.3273 & 0.3985 & 0.4382 & 0.4680 & 0.5105 & 0.5772 & 0.6598 \\
\hline
\end{tabular}

Example 1. Let $d=1, \Omega=(0,1), a(x)=x^{2}+1, c(x)=-(x+1)$. Take an initial function

$$
f(x)=x(1-x) \mathrm{e}^{x^{\alpha}} \sin 5 \pi x .
$$

In order to avoid 'inverse crime', we use a finer grid to compute the forward problem, i.e. take $M=100, N=200$ and choose $M=50, N=100$ for solving the regularized problem.

In Figure 1, we show the final data for Example 1 in the case of $\alpha=0.2,0.8$, respectively.

The numerical results by using the a posteriori parameter choice rule for various noise levels $\varepsilon=$ $0.005,0.01,0.05$ in the case of $\alpha=0.2,0.8$ are showed in Figure 2 in which we use $\mu=5.7 e-8,1.1 e-7,6.1 e-7$ for $\alpha=0.2$ and $\mu=1.4 e-8,2.9 e-8,1.5 e-7$ for $\alpha=0.8$. We can see that the numerical results are in good agreement with the exact shape.

In Table 1, we show the numerical errors of Example 1 for different $\alpha$ with a fixed $\varepsilon=0.01$, it can be seen that the numerical accuracy is very stable to the fractional order $\alpha$.

The numerical errors and convergence orders for Example $1(\alpha=0.6)$ with different $\varepsilon$ are shown in Table 2, from which we can see that the numerical error is decreasing as the level of noise becomes smaller and the convergence order is close to 0.5 . This is consistent with our convergence estimate. 


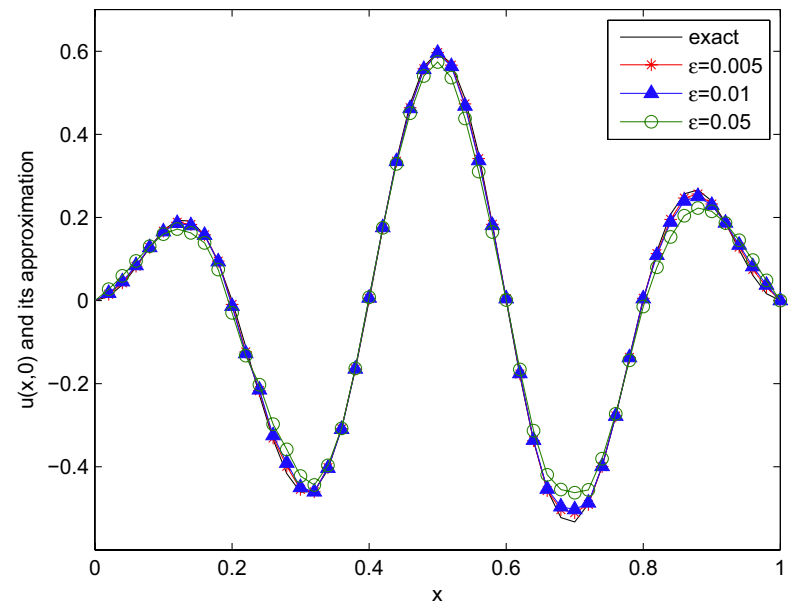

(a) $\alpha=0.2$

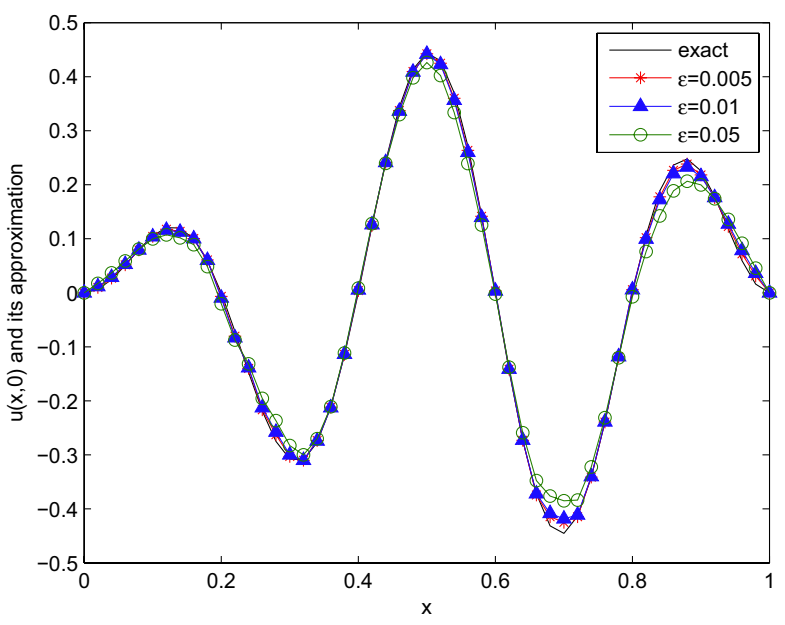

(b) $\alpha=0.8$

FiguRE 2. The exact solution and the regularized solutions by using the a posteriori parameter choice rule for Example 1.

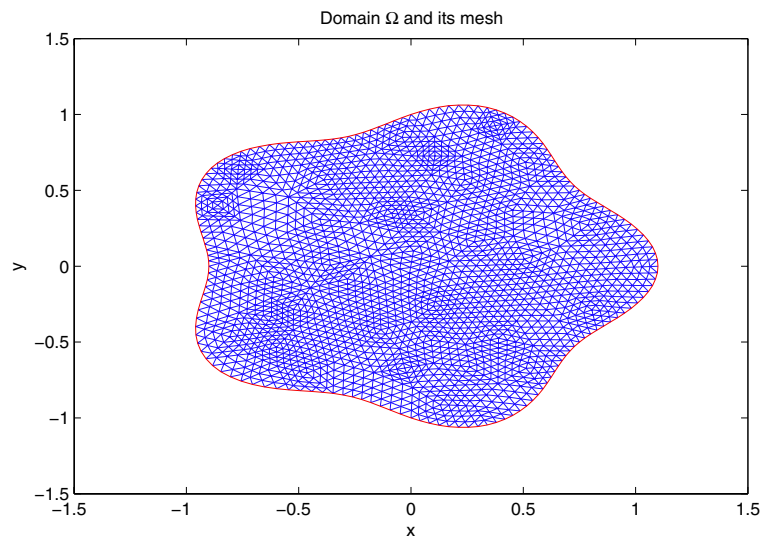

FigURE 3. Domain and mesh.

Example 2. Consider the case of $d=2$ and denote the coordinate as $(x, y)$. Let $\Omega$ be a bounded domain with a smooth boundary given by parametrization

$$
\partial \Omega=\{(x, y)=r(\theta)(\cos \theta, \sin \theta), \theta \in[0,2 \pi]\},
$$

where $r(\theta)=1+0.1 \cos 5 \theta$, see Figure 3 for the configuration of $\Omega$.

We take $a_{11}(x, y)=x^{2}+3, a_{12}(x, y)=a_{21}(x, y)=x+y+1, a_{22}(x, y)=y^{2}+3, c(x, y)=-(x+y)^{2}$ and the exact initial function is

$$
f(x, y)=\exp \left(\frac{\alpha}{\left(\frac{\sqrt{x^{2}+y^{2}}}{r(\theta)}\right)^{2}-1}\right),
$$

where $\theta$ is the polar angle of $(x, y)$. For $(x, y)=(0,0)$, we define $f(0,0)=\exp (-\alpha)$. 


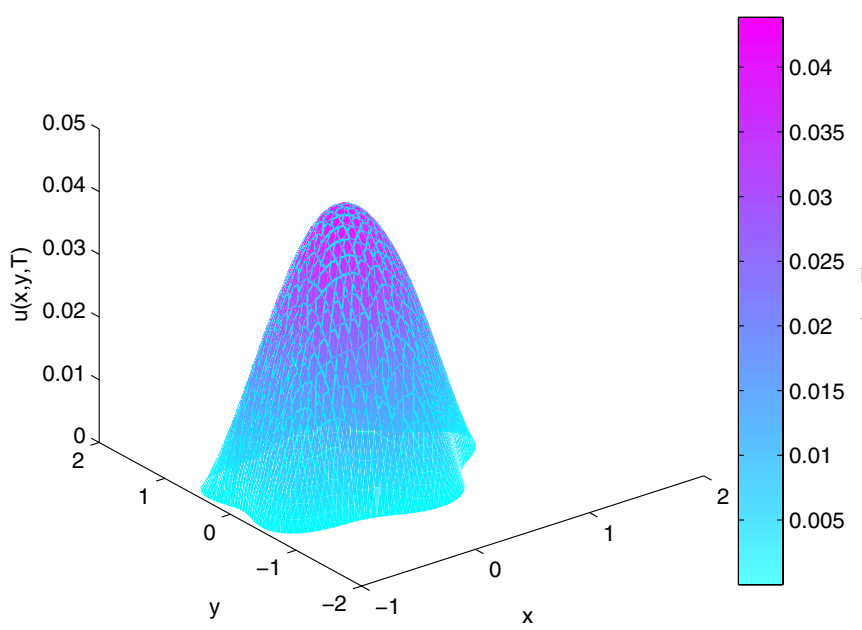

(a) $\alpha=0.2$

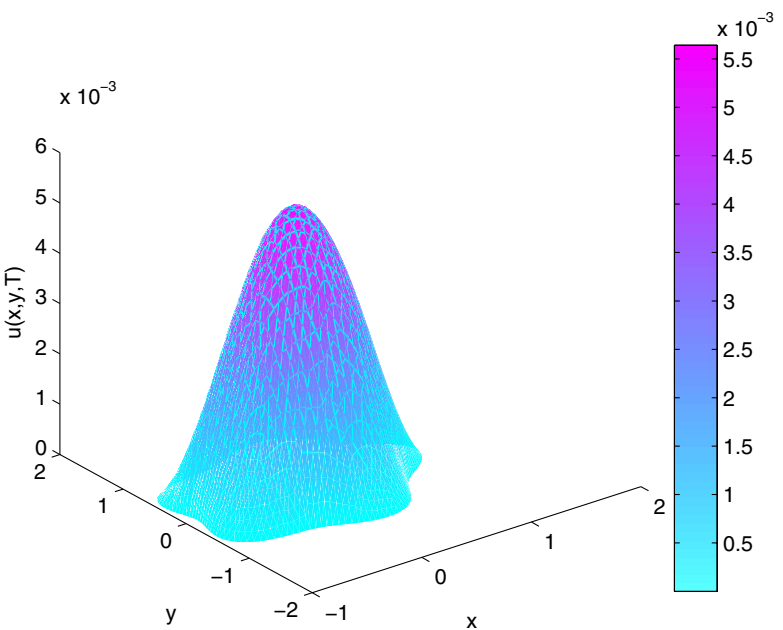

(b) $\alpha=0.8$

FiguRE 4. The final data for Example 2.

TABLE 3. Numerical results for Example 2 with various $\alpha$ and a fixed $\varepsilon=0.005$.

\begin{tabular}{ccccccc}
\hline$\alpha$ & 0.05 & 0.1 & 0.3 & 0.7 & 0.9 & 0.95 \\
\hline$e(f, 0.005)$ & 0.2067 & 0.1332 & 0.0569 & 0.0365 & 0.0290 & 0.0272 \\
$e_{r}(f, 0.005)$ & 0.1372 & 0.0991 & 0.0610 & 0.0711 & 0.0740 & 0.0741 \\
\hline
\end{tabular}

TABLE 4. Numerical results for Example 2 with $\alpha=0.6$.

\begin{tabular}{ccccccccc}
\hline$\varepsilon$ & 0.0005 & 0.001 & 0.002 & 0.004 & 0.008 & 0.016 & 0.032 & 0.064 \\
\hline$e(f, \varepsilon)$ & 0.0255 & 0.0292 & 0.0337 & 0.0387 & 0.0441 & 0.0507 & 0.0599 & 0.0741 \\
$e_{r}(f, \varepsilon)$ & 0.0431 & 0.0493 & 0.0570 & 0.0654 & 0.0746 & 0.0857 & 0.1012 & 0.1252 \\
\hline Order & & 0.1932 & 0.2101 & 0.1986 & 0.1890 & 0.2005 & 0.2392 & 0.3076 \\
\hline
\end{tabular}

In solving the forward problem, we use the matlab command pdeeig and spend 40 minutes to compute the first 338 eigenvalues in interval $[0,6000]$ and the corresponding eigenfunctions.

To obtain the regularized solution $u_{\mu}^{\delta}(x, y, 0)$, along the time direction, we take $N=20$. The mesh in domain $\Omega$ is generated by PDE toolbox in matlab and consists of 2205 nodes and 4256 triangles.

In Figure 4, we show the final data for Example 2 in the case of $\alpha=0.2,0.8$, respectively.

In Figure 5, we show the exact initial data and the regularized approximations given by the a posteriori parameter choice rule with $\varepsilon=0.01$ in the case of $\alpha=0.2,0.8$ in which we use $\mu=9.256 e-6$ for $\alpha=0.2, \mu=$ $4.6147 e-6$ for $\alpha=0.8$. It can be observed that the proposed method gives accurate numerical reconstructions and is also effective for two dimensional example.

In Table 3, we show the numerical results of Example 2 for various $\alpha$ with $\varepsilon=0.005$ from which we can see the numerical results depend very little on $\alpha$.

The numerical results and convergence orders of Example $2(\alpha=0.6)$ for different $\varepsilon$ are shown in Table 4 . We can also see that the numerical errors become smaller if decreasing amounts of random noise. The convergence order is about 0.2 which is somewhat lower than the one in Example 1. One possible reason is that Example 2 is not smooth enough as Example 1. 


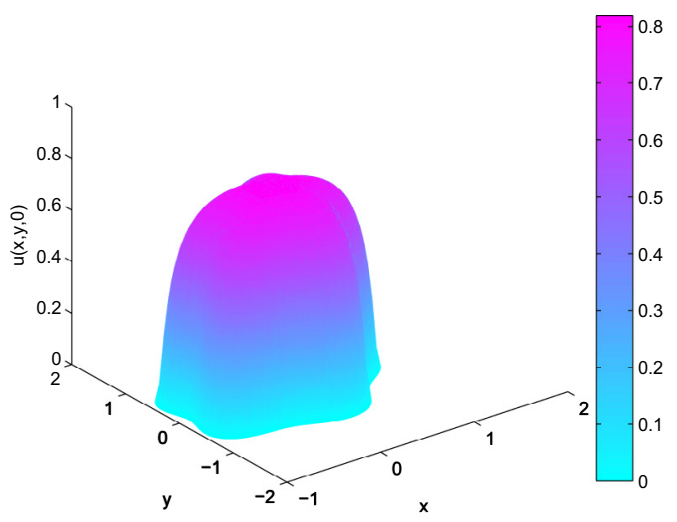

(a) Exact $u(x, y, 0)$ for $\alpha=0.2$

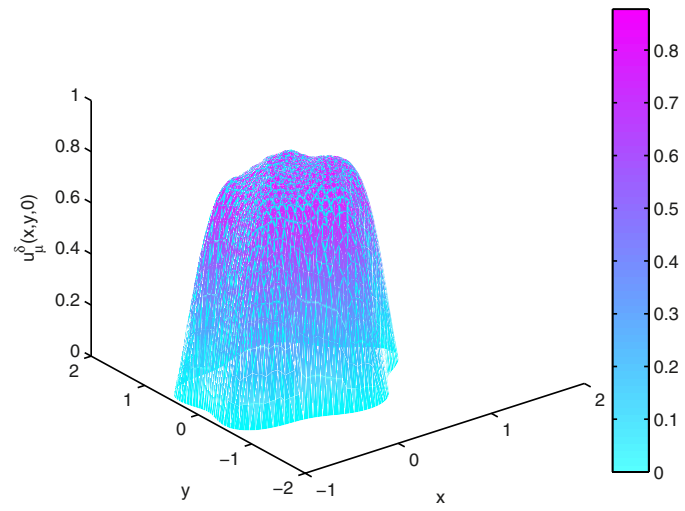

(c) Numerical $u_{\mu}^{\delta}(x, y, 0)$ for $\alpha=0.2$

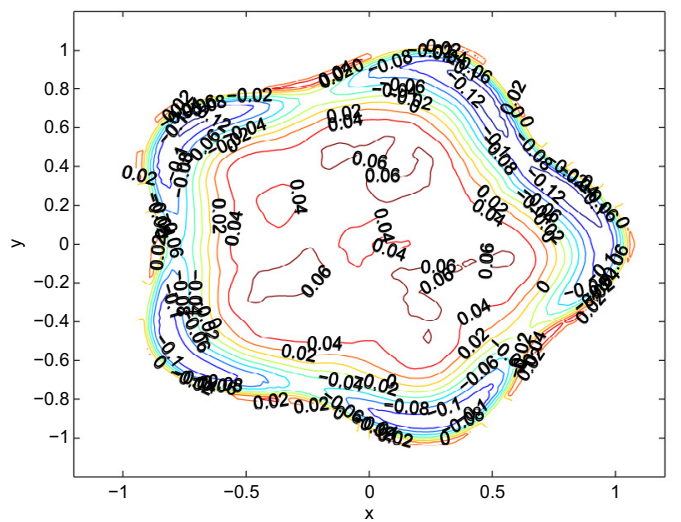

(e) Numerical error for $\alpha=0.2$

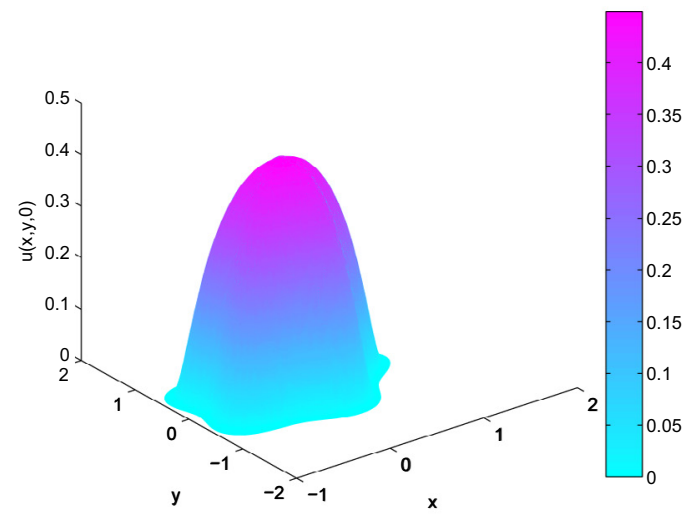

(b) Exact $u(x, y, 0)$ for $\alpha=0.8$

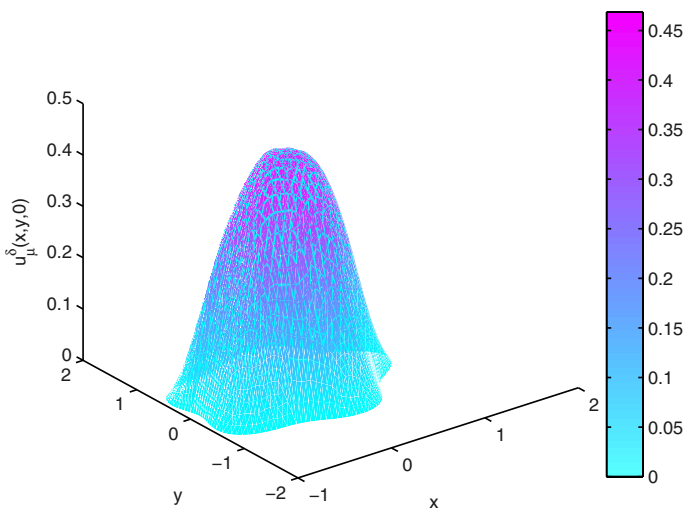

(d) Numerical $u_{\mu}^{\delta}(x, y, 0)$ for $\alpha=0.8$

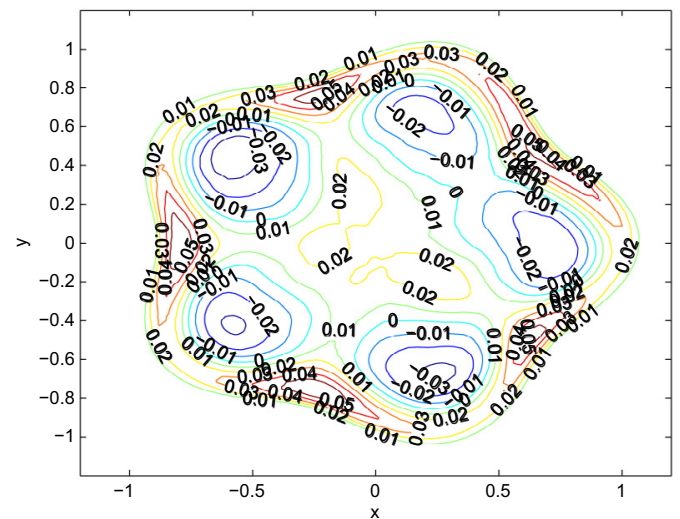

(f) Numerical error for $\quad \alpha=0.8$

FiguRE 5 . The exact and regularized solutions given by the a posteriori parameter choice rule for Example 2. 


\section{Conclusion}

In this paper, we investigate the backward problem for a time-fractional diffusion equation with variable coefficients defined in a general domain. The conditional stability is given. We propose a modified quasi-boundary value method for obtaining a regularized solution. Based on an a priori assumption for the exact solution, the error estimates are obtained under an a priori regularization parameter choice rule and Morozov's discrepancy principle, respectively. Numerical examples show that our proposed method is effective and stable.

Acknowledgements. The authors would like to thank the editor and the anonymous referees for their valuable comments and helpful suggestions that improve the quality of our paper. This work is supported by the NSF of China (11371181, 11171136) and the Fundamental Research Funds for the Central Universities (lzujbky-2013-k02).

\section{REFERENCES}

[1] K.A. Ames and J.F. Epperson, A kernel-based method for the approximate solution of backward parabolic problems. SIAM J. Numer. Anal. (1997) 1357-1390.

[2] K.A. Ames and L.E. Payne, Asymptotic behavior for two regularizations of the Cauchy problem for the backward heat equation. Math. Models Methods Appl. Sci. 8 (1998) 187.

[3] B. Berkowitz, H. Scher and S.E. Silliman, Anomalous transport in laboratory-scale, heterogeneous porous media. Water Resour. Res. 36 (2000) 149-158.

[4] J. Cheng, J. Nakagawa, M. Yamamoto and T. Yamazaki, Uniqueness in an inverse problem for a one-dimensional fractional diffusion equation. Inverse Problems 25 (2009) 115002.

[5] G. Chi, G. Li and X. Jia, Numerical inversions of a source term in the fade with a dirichlet boundary condition using final observations. Comput. Math. Appl. 62 (2011) 1619-1626.

[6] G.W. Clark and S.F. Oppenheimer, Quasireversibility methods for non-well-posed problems Electron. J. Differ. equ. (1994) $1-9$.

[7] M. Denche and K. Bessila, A modified quasi-boundary value method for ill-posed problems. J. Math. Anal. Appl. 301 (2005) $419-426$.

[8] X.L. Feng, L. Eldén and C.L. Fu, A quasi-boundary-value method for the cauchy problem for elliptic equations with nonhomogeneous neumann data. J. Inverse Ill-Posed Probl. 18 (2010) 617-645.

[9] D.N. Hào, N.V. Duc and D. Lesnic, A non-local boundary value problem method for the Cauchy problem for elliptic equations. Inverse Probl. 25 (2009) 055002.

[10] D.N. Hào, N.V. Duc and D. Lesnic, Regularization of parabolic equations backward in time by a non-local boundary value problem method. IMA J. Appl. Math. 75 (2010) 291-315.

[11] D.N. Hào, N.V. Duc and H. Sahli, A non-local boundary value problem method for parabolic equations backward in time. J. Math. Anal. Appl. 345 (2008) 805-815.

[12] Y.J. Jiang and J.T. Ma, High-order finite element methods for time-fractional partial differential equations. J. Comput. Appl. Math. 235 (2011) 3285-3290.

[13] B.T. Jin and W. Rundell, An inverse problem for a one-dimensional time-fractional diffusion problem. Inverse Probl. 28 (2012).

[14] S.M. Kirkup and M. Wadsworth, Solution of inverse diffusion problems by operator-splitting methods. Appl. Math. Modelling 26 (2002) 1003-1018.

[15] J.J. Liu and M. Yamamoto, A backward problem for the time-fractional diffusion equation. Appl. Anal. 89 (2010) $1769-1788$.

[16] Y. Luchko, Some uniqueness and existence results for the initial-boundary-value problems for the generalized time-fractional diffusion equation. Comput. Math. Appl. 59 (2010) 1766-1772.

[17] Y. Luchko, Maximum principle and its application for the time-fractional diffusion equations. Fract. Calc. Appl. Anal. 14 (2011) 110-124.

[18] F. Mainardi, G. Pagnini and R. Gorenflo, Some aspects of fractional diffusion equations of single and distributed order. Appl. Math. Comput. 187 (2007) 295-305.

[19] R. Metzler and J. Klafter, The random walk's guide to anomalous diffusion: a fractional dynamics approach. Phys. Rep. 339 (2000) $1-77$.

[20] R. Metzler and J. Klafter, Subdiffusive transport close to thermal equilibrium: From the Langevin equation to fractional diffusion. Phys. Rev. E 61 (2000) 6308-6311.

[21] D.A. Murio, Stable numerical solution of a fractional-diffusion inverse heat conduction problem. Comput. Math. Appl. 53 (2007) 1492-1501.

[22] D.A. Murio, Implicit finite difference approximation for time fractional diffusion equations. Comput. Math. Appl. 56 (2008) $1138-1145$.

[23] D.A. Murio, Time fractional IHCP with Caputo fractional derivatives. Comput. Math. Appl. 56 (2008) $2371-2381$.

[24] D.A. Murio, Stable numerical evaluation of Grünwald-Letnikov fractional derivatives applied to a fractional ihcp. Inverse Probl. Sci. Engrg. 17 (2009) 229-243. 
[25] D.A. Murio and C.E. Mejía, Source terms identification for time fractional diffusion equation. Revista Colombiana de Matemáticas 42 (2008) 25-46.

[26] D.A. Murio, Implicit finite difference approximation for time fractional diffusion equations. Comput. Math. Appl. 56 (2008) $1138-1145$

[27] I. Podlubny, Fractional differential equations, in vol. 198 of Math. Sci. Eng. Academic Press Inc., San Diego, CA (1999).

[28] I. Podlubny and M. Kacenak, Mittag-leffler function. The MATLAB routine, available at http://www.mathworks.com /matlabcentral/fileexchange (2006).

[29] H. Pollard, The completely monotonic character of the mittag-leffler function $\mathrm{E}_{\alpha}(-x)$. Bull. Amer. Math. Soc. 54 (1948) $1115-1116$

[30] Z. Qian, Optimal modified method for a fractional-diffusion inverse heat conduction problem. Inverse Probl. Sci. Engrg. 18 (2010) 521-533.

[31] W. Rundell, X. Xu and L. H. Zuo, The determination of an unknown boundary condition in a fractional diffusion equation. Appl. Anal. http://dx.doi.org/10.1080/00036811.2012.686605.

[32] K. Sakamoto and M. Yamamoto, Initial value/boundary value problems for fractional diffusion-wave equations and applications to some inverse problems. J. Math. Anal. Appl. 382 (2011) 426-447.

[33] E. Scalas, R. Gorenflo and F. Mainardi, Fractional calculus and continuous-time finance. Physica A 284 (2000) $376-384$.

[34] R. Scherer, S.L. Kalla, L. Boyadjiev and B. Al-Saqabi, Numerical treatment of fractional heat equations. Appl. Numer. Math. 58 (2008) 1212-1223.

[35] R.E. Showalter, The final value problem for evolution equations. J. Math. Anal. Appl. 47 (1974) 563-572.

[36] R.E. Showalter, Cauchy problem for hyper-parabolic partial differential equations. North-Holland Math. Stud. 110 (1985) 421-425.

[37] I.M. Sokolov and J. Klafter, From diffusion to anomalous diffusion: A century after Einsteins Brownian motion. Chaos 15 (2005) 1-7.

[38] H. Wei, W. Chen, H.G. Sun and X.C. Li, A coupled method for inverse source problem of spatial fractional anomalous diffusion equations. Inverse Probl. Sci. Engrg. 18 (2010) 945-956.

[39] W. Wyss, The fractional diffusion equation. J. Math. Phys. 27 (1986) 2782-2785.

[40] M. Yang and J.J. Liu, Solving a final value fractional diffusion problem by boundary condition regularization. Appl. Numer. Math. 66 (2013) 45-58.

[41] P. Zhang and F. Liu. Implicit difference approximation for the time fractional diffusion equation. J. Appl. Math. Comput. 22 (2006) 87-99.

[42] Y. Zhang and X. Xu, Inverse source problem for a fractional diffusion equation. Inverse Probl. 27 (2011) 035010.

[43] G.H. Zheng and T. Wei, Spectral regularization method for a Cauchy problem of the time fractional advection-dispersion equation. J. Comput. Appl. Math. 233 (2010) 2631-2640.

[44] G.H. Zheng and T. Wei, A new regularization method for a Cauchy problem of the time fractional diffusion equation. Advances Comput. Math. 36 (2012) 377-398.

[45] G.H. Zheng and T. Wei, Two regularization methods for solving a riesz-feller space-fractional backward diffusion problem. Inverse Probl. 26 (2010) 115017.

[46] P. Zhuang and F. Liu, Implicit difference approximation for the time fractional diffusion equation. J. Appl. Math. Comput. 22 (2006) 87-99. 